\title{
Requisitos de Qualidade de Usabilidade: análise da utilização em sistemas de uma instituição financeira
}

\author{
Angélica Toffano S. Calazans ${ }^{1}$, Eloisa Toffano S. Masson ${ }^{1}$, Roberto A. \\ Paldês $^{1}$, Fernando de A. Guimarães ${ }^{1}$, Kiane M. Rezende ${ }^{1}$, Ricardo \\ Ajax. D. Kosloski \\ ${ }^{1}$ Centro Universitário de Brasilia - Uniceub - Brasília - Brasil \\ angelica.calazans@uniceub.br, feloisa.masson, roberto.paldes, \\ fernando.guimaraes\} @ceub.edu.br, kianerezende@gmail.com \\ ${ }^{2}$ Universidade de Brasilia - Brasília - Brasil \\ rikosd@gmail.com
}

\begin{abstract}
Os requisitos de qualidade com foco na usabilidade estão relacionados à facilidade de uso e de aprendizado de um sistema pelo cliente, contribuindo para o desempenho na sua utilização e para a satisfação do usuário. Essas características ganham relevância pelo alcance e aprovação amplos que devem apresentar nos sistemas da área financeira. Assim, a pesquisa tem como objetivo aumentar a compreensão sobre a utilização dos requisitos de qualidade de usabilidade. Foram analisadas especificações de 26 sistemas de uma organização financeira pública de grande porte, incluindo sistemas de uso externo e interno à organização. Foi utilizada a análise de conteúdo e o software Nvivo, sendo categorizados e analisados 264 requisitos de qualidade de usabilidade. Considerando que os requisitos de qualidade de usabilidade selecionados diferiam em seu nível de abstração, detalhe e completude, foram definidos critérios para a classificação. Utilizaram-se as subcaracterísticas de Usabilidade da ISO/IEC 25010 para a classificação. Como resultado obtido, constatou-se que grande parte dos requisitos definidos atendem à norma, apesar de terem sido classificados com nomenclaturas diferentes. A análise também identificou requisitos que seguem um padrão comum. Identificaram-se, ainda, as subcaracterísticas de Usabilidade com maior interesse: apreensibilidade (37\%) e estética da interface do usuário (27\%) dos requisitos elicitados. A subcaracterística de acessibilidade obteve menor percentual de atenção, com $1,89 \%$ dos requisitos identificados.
\end{abstract}

Keywords: ISO/IEC 25000. Requisitos de qualidade. Não funcionais. Usabilidade.

\section{Introdução}

A qualidade pode influenciar todo o esforço de entrega de um sistema. $\mathrm{O}$ valor de um software encontra um primeiro apoio na precisão de seus requisitos $[1,2]$, notadamente quando se consideram os requisitos de 
qualidade o mais cedo possível [3]. A especificação consistente desses requisitos impactará outras disciplinas da Engenharia de Software, tais como o projeto e os testes [4].

Diversos autores $[5,6,7]$ denominam um requisito de qualidade como requisito não funcional (RNF). A distinção entre requisitos não funcionais e requisitos funcionais convencionou-se na Engenharia de Software, pelo contraste entre o "como" e o "quê" os sistemas realizam ou oferecem de recursos [5]. Os RNF são classificados [8] em requisitos do produto (eficiência, confiabilidade, portabilidade), requisitos organizacionais ou de processo (entrega, implementação, padrões) e externos (interoperabilidade, éticos e legais). Com uma visão ainda mais abrangente, já foram identificadas 114 terminologias para requisitos não funcionais em uma revisão sistemática sobre o assunto [9].

As normas da série ISO/IEC $25000[10,11,12,13]$ trazem uma estrutura para a aplicação, controle e avaliação da qualidade do software. São denominadas System and Software Quality Requirements and Evaluation (SQuaRE). Elas realizam uma junção e evolução das regras vigentes nas ISO/IEC 9126 e 14598 [14, 15], descontinuadas após a publicação desta série. As SQuaRE não apresentam a terminologia requisito não funcional. De acordo com ISO 25030 [13], os requisitos de um produto de software se classificam em requisitos inerentes (funcionais e de qualidade) e requisitos atribuídos (preço,data entrega etc).

O contexto da pesquisa é a aplicação das normas ISO/IEC 25000 no setor de desenvolvimento de sistemas de uma instituição financeira pública brasileira com alcance nacional. Considerou-se que as citadas normas são um significante modelo e ponderou-se também o perfil da organização, já que a característica de usabilidade foi destacada pelos seus analistas como um fator crítico. Na literatura [4], a usabilidade também é reconhecida como crítica, pois tem um impacto positivo sobre a decisão dos indivíduos de utilizar o software.

Dessa forma, estabeleceram-se as seguintes questões de pesquisa:

- Como a classificação dos requisitos de qualidade, com foco na usabilidade, é utilizada na prática pelos desenvolvedores da organização?

- Existe relação dos requisitos de qualidade de usabilidade da ISO/IEC 25010, considerando suas subcategorias, com a prática de elicitação /documentação de requisitos da organização?

- Quais subcategorias dos resquisitos de usabilidade têm maior representatividade, nos diferentes domínios de aplicação? 
A relevância da investigação empírica está em identificar os aspectos da especificação de requisitos a partir de um referencial teórico contemporâneo e de uma abordagem prática. Cada vez mais, as organizações precisam se preocupar em como os requisitos de qualidade de usabilidade são tratados, implementados e testados [4], [16].

Dessa forma, os objetivos específicos do presente trabalho são: compreender como, na prática, o universo considerado classifica e documenta os requisitos de qualidade de usabilidade; entender como a prática de uma organização se relaciona com as subcaracterísticas de usabilidade das normas ISO/IEC 25010, nos seus produtos de software; e, compreender qual a importância atribuída às subcaracterísticas de qualidade relacionadas à usabilidade.

Assim, a segunda seção do artigo sintetiza as características da séria ISO 25000 e a terceira sobre outros trabalhos relacionados à usabilidade. A seção 4 apresenta o método da investigação realizada. Em seguida, são listados os resultados obtidos e realizada a sua interpretação. Por fim, são expostas as conclusões do trabalho.

\section{A série ISO/IEC 25000}

A ISO/IEC 25000:2014 [15] é uma norma técnica que orienta o uso da série de normas internacionais e propõe cinco divisões: a de Gestão da Qualidade (2500n), a de Modelo de Qualidade (2501n), a de Medição da Qualidade (2502n), a de Requisitos de Qualidade (2503n) e a de Avaliação da Qualidade (2504n).

A divisão 2503n é composta pela norma ISO / IEC 25030 que fornece orientações para o processo de elicitação de requisitos de qualidade para um produto de software a ser desenvolvido ou como entrada para um processo de avaliação [17]. Os requisitos de qualidade de software incluem requisitos de qualidade em uso, requisitos de qualidade externa e requisistos de qualidade interna, conforme destacado na Fig. 1.

Compondo a divisão 2501n, a norma 25010 [10] define modelos de qualidade para o uso e para os produtos de software. Entre os atributos da qualidade de uso de um software, está a sua capacidade de minimizar ou evitar riscos tanto aos usuários quanto à organização, ou ao próprio projeto [18]. Já a qualidade dos produtos deve seguir o modelo formado por oito características: Adequação Funcional, Performance Eficiente, Compatibilidade, Usabilidade, Confiabilidade, Segurança, Manutenibilidade e Portabilidade. 


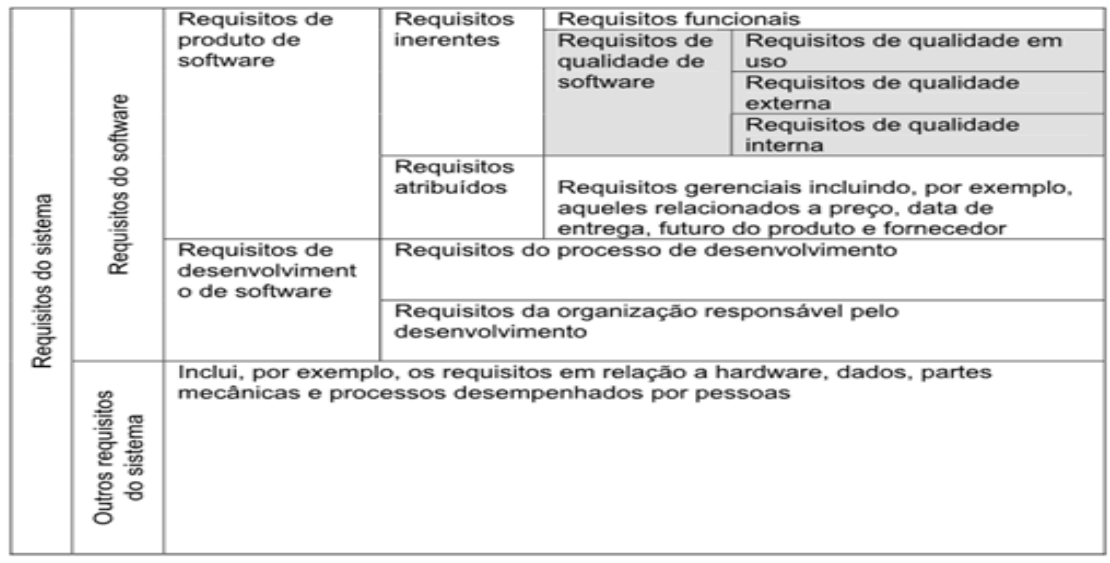

Fig. 1. Classificação dos requisitos de um sistema Fonte: [13].

A caraterística de Usabilidade é dividida em seis outras subcaracterísticas:

- Acessibilidade (Acessibility): possibilidade de utilização do software por pessoas com características e capacidades diferenciadas.

- Apreensibilidade (Learnability): grau em que o produto possibilita ao usuário aprender a usá-lo com eficiência, eficácia, satisfação e livre de riscos.

- Estética da interface do usuário (User interface aesthetics): grau em que uma interface permite uma interação agradável e satisfatória para o usuário.

- Operabilidade (Operability): grau em que o produto é fácil de operar, controlar e apropriado ao uso.

- Reconhecimento apropriado (Appropriateness recognisability): grau que os usuários conseguem compreender se o produto é adequado às suas necessidades.

- Proteção contra erros do usuário (User error protection): grau em que o produto contribui para que erros sejam evitados e tratados durante a sua utilização.

\section{Trabalhos sobre usabilidade}

O conceito inicial de usabilidade se relacionava com a maneira que um sistema emprega para atingir suas metas com eficácia, eficiência e 
satisfação em um contexto específico de uso [19]. O conceito foi estendido para incluir experiência do usuário e o sucesso percebido [18].

Um dos requisitos de qualidade mais dicutidos é a usabilidade [16]. Uma pesquisa constatou [20] que um dos três principais critérios que conduziram ao desenvolvimento cada vez maior de aplicações Web foi a usabilidade. Outro estudo realizado em 2016 [16] analisou métodos, procedimentos, ferramentas e metodologias de validação empírica empregadas em trabalhos de avaliação de requisitos não funcionais de qualidade e seus propósitos, e apontou o crescente interesse pelo requisito de usabilidade.

Já outro estudo empírico [21] foi realizado sobre 21,7 milhões de postagens e 32,5 milhões de comentários do Stack Overflow, um website de perguntas e respostas entre especialistas em programação. Identificou-se que os desenvolvedores discutem principalmente usabilidade e confiabilidade. $\mathrm{O}$ estudo sinaliza que se deve concentrar na usabilidade para superar dificuldades de manutenção.

Apesar de não focar somente o contexto da Usabilidade, o trabalho de [5] se destaca, pois faz uma avaliação de 530 requisitos não funcionais de várias indústrias (utilizando a ISO 9126) e classifca-os, considerando uma nova proposta, em requisitos de comportamento e representacionais.

\section{$4 \quad$ Método}

\subsection{Objeto de estudo}

Os objetos de estudo usados para responder as perguntas de pesquisa, constituem-se de documentos de especificações industriais de requisitos de qualidade de uma organização financeira governamental de grande porte. A organização possui um setor próprio de desenvolvimento com sítios em três cidades brasileiras, desenvolvendo software para atendimento de suas demandas, além de utilizar serviços de terceirização. A organização comercializa produtos e serviços ligados à habitação, financiamento de saneamento básico e infraestrutura urbana, além da gestão dos canais parceiros.

As diferentes equipes seguem padrões de desenvolvimento da organização e a metodologia utilizada é baseada no Rational Unified Process [22]. A empresa recebeu certificação MPS BR nível F em 05/2012. Os requisitos de qualidade são registrados como Especificação Suplementar. 
Inicialmente, foram coletados documentos de especificações suplementares de 211 sistemas ativos da organização analisada. Foram analisados diferentes domínios de aplicação que abrangiam sistemas on-line, batch e híbridos. Foram aplicados os seguintes filtros:

- Selecionados os documentos de especificações no período de 2007 a 2018 (10 anos);

- Selecionados somente os sistemas on-line (com o objetivo de analisar escopo similar);

- Excluídos os sistemas BI, uma vez que a estes utilizam uma ferramenta distinta dos usuais documentos de especificação;

- Excluídos os sistemas cuja especificação não estava acessível.

Foram selecionados ao final, 26 sistemas e a Tabela 1 apresenta o resumo dos objetos de estudo classificados por domínio de aplicação.

Tabela 1. Domínios de aplicação analisados

\begin{tabular}{lll}
\hline Domínios de Aplicação & Qtd de sistemas & \% participação \\
\hline Administrativos e cadastro & 11 & 42,31 \\
Canais de atendimento & 4 & 15,38 \\
Cartões & 3 & 11,54 \\
Controladoria & 2 & 7,69 \\
Emprestar e financiar & 1 & 3,85 \\
Financeiro e Controladoria & 1 & 3,85 \\
Habitação & 1 & 3,85 \\
Infra tecnológica e geoprocessamento & 2 & 7,69 \\
Portal de conteúdo & 1 & 3,85 \\
Total & 26 & 100 \\
\hline
\end{tabular}

Fonte: Autores

\subsection{Procedimentos de preparação e coleta de dados}

Para responder as perguntas de pesquisa, foram selecionados os requisitos de qualidade de Usabilidade e em seguida, realizou-se uma classificação e análise. O procedimento foi realizado pelos dois primeiros autores em par, ambos com experiência em pesquisa em engenharia de requisitos.

Foi utilizado o software Nvivo [23] para análise e classificação dos dados. É um software que suporta vários métodos qualitativos de pesquisa de diversas fontes de informação.

Foi identificado que os requisitos de qualidade de Usabilidade selecionados diferiam em seu nível de abstração, detalhe e completude. Por 
tanto, foram processados cada um deles da seguinte forma:

- Interpretação completa: considerou-se o requisito de qualidade como estava escrito e classificados no grupo da Usabilidade da organização.

- Sub interpretação: foi considerada apenas uma parte dos requisitos de qualidade - relacionados claramente como característica de usabilidade [10], [13] e se desconsiderou o resto do requisito.

- Requisito de divisão: foi dividido o requisito de Usabilidade em um conjunto de requisitos porque o requisito original abordou mais de uma propriedade desejada de um sistema.

- Excluir do estudo: foram excluídos os requisitos de qualidade que, apesar de estarem apresentados como Usabilidade, não pertenciam ao escopo estudado (por exemplo, conformidade, portabilidade), ou se não fosse claro o entendimento do requisito, por informações vagas etc.

Do total de 308 requisitos, foram excluídos 44 (14\%), que foram classificados nos itens Conformidade, Diversos e Portabilidade. Foram classificados 264 requisitos, seguindo o padrão [12] para Usabilidade.

\section{$5 \quad$ Resultados e análise dos dados}

Para responder à Questão 1, foi identificado que o documento Especificações Suplementares, da organização analisada, possui a descrição de todos os requisitos de qualidade (chamados de requisitos não funcionais) e dos requisitos condicionantes. Os requisitos de qualidade e condicionantes estão escritos em linguagem natural. Esse procedimento é coerente com outros trabalhos [5].

Os requisitos condicionantes são considerados pela organização como requisitos que especificam ou restringem o design, implementação, interface e características físicas do software. Os requisitos condicionantes são aprovados pela equipe de TI responsável pelo serviço.

A ISO/IEC 25030 identifica os requisitos funcionais, de qualidade e outros conforme Fig.1. Assim, foi possível inferir que os requisitos condicionantes estão alinhados aos requisitos de desenvolvimento de software - requisitos da organização responsável pelo desenvolvimento e outros requisitos do sistema.

A organização analisada classifica as subcategorias de Usabilidade em seis grandes grupos: Acessibilidade, Estética, Coerência da interface do usuário, Idioma, Ajuda em tempo real e Documentação do sistema. 
Essas classificações não seguem totalmente nenhum modelo específico citado anteriormente [8], [13].

Para responder à Questão 2, foram analisados detalhadamente os requisitos de qualidade de Usabilidade e classificados considerando as subcaracterísticas [12].

Apesar da existência de subcaracterística com o mesmo nome (Acessibilidade) na organização, nem todos os requisitos identificados nessa categoria foram classificados na referida subcaracterística da ISO. Alguns foram reclassificados em outra subcategoria. Por exemplo: "Disponibilizar interfaces de fácil navegabilidade" estava vinculado à Acessibilidade pela organização. Acessibilidade, pela classificação da ISO, está relacionada à utilização do software por pessoas com características e capacidades diferenciadas (deficiências, faixa etária etc). Assim, esses requisitos foram classificados para Estética da interface do usuário.

Foram encontrados casos em que requisitos semelhantes eram classificados em grupos diferentes, dependendo dos sistemas. Isso também foi corrigido. A Tabela 2 apresenta a subcaracterística, a quantidade de sistemas e a quantidade de requisitos identificados.

Tabela 2. Quantitativo de requisitos por subcaracterística de Usabilidade

\begin{tabular}{lllll}
\hline Subcaracterísticas de Usabilidade [12] & $\begin{array}{l}\text { Qtd Sis- } \\
\text { temas }\end{array}$ & $\begin{array}{l}\text { \% sobre 26 } \\
\text { sistemas }\end{array}$ & $\begin{array}{l}\text { Qtd Re- } \\
\text { quisitos }\end{array}$ & $\begin{array}{l}\text { \% sobre 264 } \\
\text { Requisitos }\end{array}$ \\
\hline Acessibilidade - Accessibility & 4 & 15,38 & 5 & 1,89 \\
Apreensibilidade - Learnability & 26 & 100,00 & 98 & 37,12 \\
$\begin{array}{l}\text { Estética da interface do usuário -User inter- } \\
\text { face aesthetics }\end{array}$ & 16 & 61,54 & 72 & 27,27 \\
$\begin{array}{l}\text { Operacionalidade - Operability } \\
\begin{array}{l}\text { Proteção contra erros do usuário -User error } \\
\text { protection }\end{array}\end{array}$ & 9 & 34,62 & 20 & 7,58 \\
$\begin{array}{l}\text { Reconhecimento apropriado - Appropriate- } \\
\text { ness recognisability }\end{array}$ & 21 & 34,62 & 25 & 9,47 \\
\hline
\end{tabular}

Fonte: Autores

Isso permite identificar que a maior parte dos requisitos de qualidade da organização relaciona-se às subcaracterísticas da norma [12], quando o foco é a característica Usabilidade, apesar de não estarem descritos com a mesma nomenclatura. Permite inferir uma preocupação da organização com relação à Usabilidade e aos padrões internacionais .

Com relação aos requisitos excluídos (14\%), identificou-se que aproximadamente $12,99 \%$ era de requisitos relacionados á Conformidade. Eles estão distribuídos em $88 \%$ dos sistemas. É interessante ressaltar que 
a Conformidade era uma subcaracterística de Usabilidade na norma anterior [14] e correspondia à adequação a padrões, etc. Pode-se inferir a importância dessa subcaracterística para uma organização de grande porte. Na nova série [24], essa subcaracterística foi eliminada. Na análise efetuada, foram classificados neste tópico, requisitos como: "Utilização dos padrões (cores, fontes, disposição de elementos) segundo norma da Organização", "O idioma a ser utilizado na interface será o Português", etc.

Com relação à Portabilidade $(0,97 \%)$, identificou-se que requisitos como "O sistema é desenvolvido para ser acessado via WEB com os principais navegadores disponíveis no mercado (Internet Explorer $10 \mathrm{e}$ Mozilla Firefox 38.1.0)" são requisitos relacionados à Característica de Portabilidade e não à Usabilidade. Assim sendo, optou-se por considerálos sem classificação para o contexto da pesquisa.

Durante a análise, foi possível identificar ainda vários requisitos seguindo um padrão comum, por exemplo: "interface suficientemente amigável", "facilitar o aprendizado" entre outras. A Tabela 3 apresenta uma análise quantitativa dos achados mais representativos. Esse resultado permite inferir que aproximadamente $30 \%$ dos requisitos analisados fazem parte de um padrão comum na organização analisada. Pesquisa [5] também identificou padrões comuns nos requisitos funcionais e não funcionais analisados.

A norma [13] cita que "os requisitos de qualidade de software devem ser verificáveis". As Fig. 2 e 3 apresentam a composição dos termos mais representativos da Tabela 03, exceto o termo "Web". Os termos

Tabela 3. Alguns padrões comuns identificados nos requisitos

\begin{tabular}{lllll}
\hline Termos comuns & $\begin{array}{l}\text { Qtd Sis- } \\
\text { temas }\end{array}$ & $\begin{array}{l}\text { \% com re- } \\
\text { lação aos } \\
26 \text { sistemas }\end{array}$ & $\begin{array}{l}\text { Qtd Re- } \\
\text { quisitos }\end{array}$ & $\begin{array}{l}\text { \% com rela- } \\
\text { ção aos 264 } \\
\text { requisitos }\end{array}$ \\
\hline Facilitar o aprendizado & 10 & 38,46 & 10 & 3,79 \\
Melhores práticas & 3 & 11,54 & 6 & 2,27 \\
Navegação e/ou interfaces amigáveis & 13 & 50,00 & 15 & 5,68 \\
Otimizar o tempo & 10 & 38,46 & 12 & 4,55 \\
Reduzir o esforço necessário & 9 & 34,62 & 9 & 3,41 \\
Seqüência lógica & 8 & 30,77 & 10 & 3,79 \\
Web (interface, plataforma, padrão) & 11 & 42,31 & 11 & 4,17 \\
\hline
\end{tabular}


"suficientemente amigáveis" e "otimizar o tempo" apresentam certa dificuldade de verificação. $\mathrm{O}$ documento disponibilizado pela organização não explicita como essa verificação é feita. A falta de ferramentas, métodos, técnicas, definição de testes são aspectos que influenciam negativamente a definição, implementação e testes dos requisitos não funcionais [25].

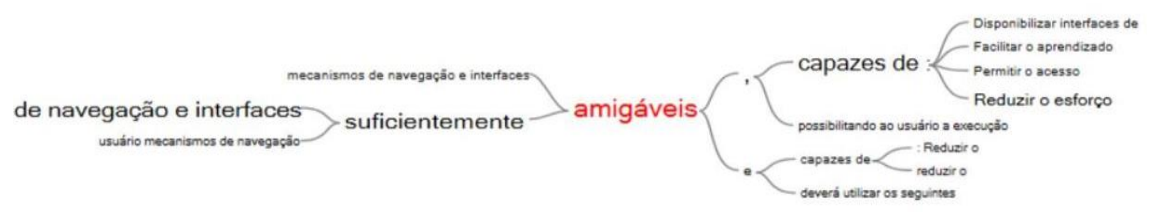

Fig. 2. Termo padrão "Suficientemente e/ou amigáveis"

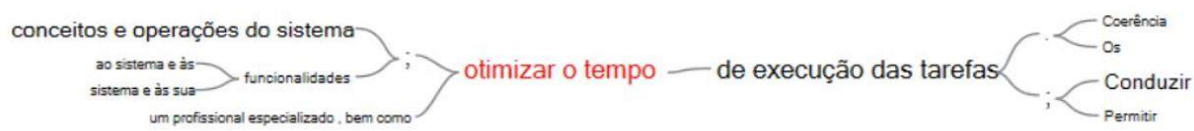

Fig. 3. Termo padrão "otimizar o tempo

Fonte: Autores

Para responder à Questão 3, a Tabela 2 apresenta os dados por subcaracterística da ISO considerando a quantidade de sistemas e o quantitativo de requisitos identificados.

Identificou-se que os requisitos relacionados à subcaracterística Apreensibilidade foram citados em todos os sistemas analisados. Além disso, essa subcaracterística em quantidade de requisitos foi também a mais referenciada, com aproximadamente $37 \%$ dos requisitos. Já a subcaracterística Reconhecimento Apropriado ficou em segundo lugar, estando incluída em aproximadamente $81 \%$ dos sistemas. Aproximadamente $17 \%$ dos requisitos estão relacionados à essa subcaracterística.

A subcaracterística Estética da interface do usuário foi a terceira mais referenciada, por aproximadamente $61 \%$ dos sistemas e consta em aproximadamente $27 \%$ dos requisitos analisados. Já as subcaracterísticas Operabilidade e Proteção contra erros do usuário constam em nove sistemas cada uma (aprox 35\%). De 8\% e 10\% dos requisitos identificados estão relacionados a estas subcaracterísticas, respectivamente.

Por último e com pouca representatividade, está classificada a Acessibilidade, que só é citada em quatro sistemas, com cinco requisitos. 
Esses resultados diferem dos resultados obtidos anteriormente [5], que identificaram em sua pesquisa, um maior quantitativo de requisitos não funcionais relacionados a Operabilidade. É interessante ressaltar que a pesquisa [5], trabalhou com vários tipos de software (embarcado, híbrido, etc), domínios e organizações. Enquanto que o trabalho atual, focou somente em sistemas on line de vários domínios (mas predominantemente sistemas internos) de uma única organização. Isso pode justificar a diferença de resultados obtidos.

A Tabela 4 apresenta a distribuição das subcaracterísticas e dos requisitos de Usabilidade por domínio de aplicação. Aproximadamente, 47\% dos requisitos analisados correspondiam a sistemas Administrativos e Cadastro (sistemas internos), enquanto que $14 \%$ relacionavam-se a sistemas de Controladoria (sistemas internos). Assim, pode-se inferir que o baixo quantitativo de requisitos de Acessibilidade relaciona-se ao quantitativo de sistemas analisados com predominância de sistemas internos na organização. Além disso, a ISO 9126 não identificava a característica acessibilidade como característica de qualidade, isso só foi implementado na série 25000 .

Tabela 4. Quantidade de subcaracterísticas e requisitos por domínio

\begin{tabular}{llll}
\hline Domínios & $\begin{array}{l}\Sigma \text { Subca- } \\
\text { racterísti- } \\
\text { cas }\end{array}$ & $\begin{array}{l}\Sigma \text { Qtd re- } \\
\text { quisitos }\end{array}$ & $\begin{array}{l}\% \text { sobre } \\
264 \text { re- } \\
\text { quisitos }\end{array}$ \\
\hline Administrativo e cadastro & 41 & 124 & 46,97 \\
Canais de atendimento & 9 & 14 & 5,30 \\
Cartões & 12 & 35 & 13,26 \\
Controladoria & 8 & 38 & 14,39 \\
Emprestar e financiar & 2 & 7 & 2,65 \\
Financeiro e controladoria & 2 & 6 & 2,27 \\
Habitação & 4 & 13 & 4,92 \\
Infra e geoprocessamento & 6 & 22 & 8,33 \\
Portal de conteúdo & 2 & 5 & 1,89 \\
Total Geral & 86 & 264 & \\
\hline
\end{tabular}

Fonte: Autores

Pode-se também inferir que os resultados obtidos na pesquisa estão influenciados pelos domínios relacionados aos sistemas internos. Essa afirmativa é parcialmente rafiticada, pois em pesquisa [5] com requisitos funcionais e não funcionais, foi identificado que a diferença de domínio tem um impacto representativo no quantitativo de requisitos [14]. 


\subsection{Ameaças à validade}

A seguir, discutem-se as ameaças à validade e as medidas adotadas para a sua mitigação.

- Problema de representatividade dos dados: os requisitos de qualidade analisados abrangem o contexto industrial selecionado. Além disso, a maior parte dos sistemas analisados pertence ao domínio de administração e cadastro (sistemas internos). Não há como mitigar essas ameaças, mas deve ser considerado que o quantitativo de sistemas e requisitos foi suficiente para se obter conclusões consistentes. A relação com outros trabalhos pode fortalecer os resultados.

- Problema de seleção dos requisitos: foram coletados os requisitos explicitamente rotulados como não funcional e de qualidade relacionados à usabilidade. Alguns requisitos de qualidade relevantes podem estar ausentes e outros irrelevantes, podem ter sido considerados. Para mitigar esse problema, estão sendo realizados estudos considerando todos os requisitos de qualidade, inclusive os condicionantes.

- Problema de preparação: foram excluídos os requisitos de qualidade que não estavam no escopo do estudo e os que não foram possíveis de compreender (informações perdidas ou vagas). Pelo pequeno percentual de excluídos, não se considera que esse procedimento comprometa a validade das conclusões.

- Problema de classificação: foi realizada a classificação por pares de pesquisadores que discutiram cada requisito de qualidade.

- Problema de contextualização: a clareza dos contextos é limitada pela necessidade de anonimato da organização e dos seus dados. Para mitigar essa ameaça, as informações privadas foram reduzidas.

\section{Conclusões e trabalhos futuros}

O objetivo dessa pesquisa foi aumentar a compreensão sobre a especificação, por uma organização financeira, dos requisitos de qualidade de usabilidade. Foram analisadas especificações de requisitos de qualidade de 26 sistemas de uma organização pública de grande porte, com emprego de análise de conteúdo e o software Nvivo.

Utilizaram-se as subcaracterísticas de Usabilidade da ISO 25010 para a classificação e um dos achados é que grande parte dos requisitos defi- 
nidos atendem à norma, apesar de terem sido classificados com nomenclaturas diferentes. Isso mostra a preocupação da organização em seguir, pelo menos em parte, alguma norma internacional. Permite também inferir que a utilização dos termos citados pela ISO, ou outro modelo, talvez não seja clara para organização e os seus desenvolvedores. Alguns conceitos, tais como "Apreensibilidade" e "Reconhecimento apropriado" podem gerar dúvidas quanto à sua aplicação.

Constatou-se que o domínio da aplicação pode influenciar os quantitativos dos requisitos de qualidade de usabilidade relacionados à cada subcaracterística e se identificaram padrões comuns de requisitos de qualidade de usabilidade.

Além disso, foi possível identificar as subcaracterísticas de Usabilidade com maior importância: Apreensibilidade e Estética da interface do usuário, com aproximadamente $37 \%$ e $27 \%$ dos requisitos, respectivamente. A subcaracterística Acessibilidade obteve menor percentual de importância com 1,89\% dos requisitos, permitindo inferir que esse baixo percentual talvez esteja relacionado ao quantitativo de sistemas internos.

Como trabalhos futuros, espera-se investigar os outros requisitos de qualidade da mesma organização buscando efetuar um comparativo com outras características. Da mesma forma, deve-se investigar como outras organizações documentam seus requisitos de qualidade, de forma a ratificar ou retificar os achados encontrados. Outro trabalho futuro é investigar por que essa organização utiliza outros termos que não a nomenclatura da ISO ou outra adotada pela indústria e academia.

\section{Referências}

1. Vilela, J., Figueiredo, B., Castro, J., Soares, M.. Goncalves, E .: Usability and Software Architecture: A Literature Review. In: IX Brazilian Symposium on Components, Architectures and Reuse Software (SBCARS), Belo Horizonte, pp. 80-89 (2015).

2. Gastaldo, D. L., Midorikawa, E. T.: Processo de Engenharia de Requisitos Aplicado a Requisitos Não-Funcionais de Desempenho: Um Estudo de Caso. In: Workshop em Engenharia de Requisitos. Piracicaba, p.302-316 (2003).

3. Cysneiros, L.M., Leite, J.C.S.P.: Using UML to Reflect Non-Functional Requirements. In: Proceedings of the CASCON 2001, Centre for Advanced Studies on Collaborative Research, November 5-7, Toronto, Ontario, Canada (2001).

4. Benslimane, Y., Cysneiros, L.M., Bahli, B. Assessing Critical functional and Non-functional Requirements for Web-based Procurement Systems: a comprehensive survey. Requirements Engineering, vol. 12, pp. 191-198 (2007).

5. Eckhardt, J., Vogelsang, A., Fernández, D.M.: Are "Non-functional" Requirements really Non-functional? An Investigation of Non-functional Requirements in Practice. In: 2016 
IEEE/ACM 38th International Conference on Software Engineering (ICSE), Austin, TX, 2016, pp. 832-842 (2016).

6. Cysneiros, L.M.; Leite, J.C.S.P.: Definindo Requisitos Não Funcionais. In: Anais do XI Simpósio Brasileiro de Engenharia de Software, pp. 49-54 (1997).

7. Domingues, J.C.S., Dapena, P.R.: Verificación de los requisitos no funcionales en el software crítico". Forum Calidad, n. 142, pp. 25-31 (2003).

8. Sommerville, I.: Engenharia Software, 8. ed. São Paulo: Pearson Addison-Wesley (2007).

9. Mairiza, D., Zowghi, D., Nurmuliani, N.: An investigation into the notion of non-functional requirements. In: SAC '10 Proceedings of the 2010 ACM Symposium on Applied Computing, pp. 311-317 (2010).

10. ISO/IEC 25010.: Systems and software engineering - Systems and software product Quality equirements and Evaluation (SQuaRE) - System and software quality models (2011).

11. ISO/IEC FDIS 25022.: Systems and software engineering - Systems and software Quality Requirements and Evaluation (SQuaRE) - Measurement of quality in use (2016).

12. ISO/IEC FDIS 25023: Systems and software engineering - Systems and software Quality Requirements and Evaluation (SQuaRE) - Measurement of system and software product quality (2016).

13. ISO/IEC. ABNT NBR ISO/IEC 25030.: Software engineering - Software product Quality Requirements and Evaluation (SQuaRE) - Quality requirements. s.l. : ISO, 2008.

14. ISO/IEC 9126: Software Engineering - Product Quality, parts 1, 2 and 3, International Organization for Standarization, Geneve (2001).

15. ISO/IEC 14598-1: Software Engineering - Product evaluation - Part 1: General, International Organization for Standarization, Geneve (2001).

16. Garcia, I., Rodrígues, I., Ahmad, M.: Evaluation of the Non-functional Requirements, of Usability: A Systematic Study. In: International Journal of Advanced Research in Computer Sciense, volume 3, número 3, Maio/Junho (2016).

17. ISO 25000 Homepage, http://iso25000.com/index.php/en/iso-25000-standards, último acesso em 28/06/2018.

18. Bevan, N., Carter, J., Earthy, J., Geis, T., Harker, S.: New ISO Standards for Usability, Usability Reports and Usability Measures. M. Kurosu (Ed.): HCI 2016, Part I, LNCS 9731, pp. 268-278 (2016).

19. ISO 9241-11: Ergonomic requirements for office work with visual display terminals (VDTs) - Part 11 Guidance on usability (1998).

20. Benslimane, Y., Cysneiros, L.M. , Bahli, B. (2007).: Assessing critical functional and nonfunctional requirements for web-based procurement systems: a comprehensive survey. Requirements Engineering. 12, 191-198 (2007).

21. Zou, J., Xu, L., Yang, M., Zhang, X., Yang, D.: Towards comprehending the non-functional requirements through developers' eyes: An exploration of stack' overflow using topic analysis. Information and Software Technology, vol. 84, pp. 19-32 (2017).

22. Kroll, P., Kruchten, P.: The rational unified process made easy: a practitioner's guide to the RUP. Addison-Wesley Professional (2003).

23. Nvivo Suite, http://www.qsrinternational.com/nvivo-portuguese, último acesso em $14 / 06 / 2018$

24. ISO: ISO/IEC 25000:2014 Systems and software engineering - Systems and software Quality Requirements and Evaluation (SQuaRE) - Guide to SQuaREtle (2014).

25. Moreno, J. C., Marciszack, M. Martín.: La Usabilidad Desde La Perspectiva De La Validación de Requerimientos No Funcionales Para Aplicaciones Web. 1er Congreso CoNaIISI 2013. Universidad Tecnológica nacional - Facultad Regional Córdoba (2013). 